\title{
Necesidades de la investigación turística en México REFLEXIONES EN EL MARCO DE LA INVESTIGACIÓN INTERNACIONAL
}

\author{
Carlos Monterrubio \\ Universidad Autónoma del Estado de México \\ jcmonterrubioc@uaemex.mx
}

El análisis de la investigación turística internacional permite conocer los avances en torno a la construcción global del conocimiento en el campo y con ello determinar necesidades y oportunidades de investigación en contextos nacionales específicos. El objetivo de este artículo es identificar necesidades de investigación respecto al estado que guarda el turismo como objeto de estudio en México, a partir del análisis de los avances reportados en la investigación turística internacional y nacional. El análisis postula cinco necesidades de investigación en torno al estado que guardan los estudios turísticos en el país: ampliar la cobertura temática reconociendo al turismo como ente multidimensional; estudiar las tendencias de los avances a partir de metodologías utilizadas en otros contextos internacionales; analizar el estado de los diferentes tópicos específicos hasta hoy abordados; identificar el liderazgo de autores y revistas en México, e incorporar el conocimiento nacional a los debates internacionales del turismo.

Palabras clave: Investigación turística, conocimiento turístico, tendencias, revistas científicas en turismo, México.

\section{Requirements for tourism ReSEARCH in MeXico: REFLECTIONS WITHIN THE INTERNATIONAL RESEARCH FRAMEWORK}

The study of international tourism research is useful in understanding the progress in the global construction of knowledge in the field. It can help to identify research needs and opportunities both at the global and national levels. The aim of this paper is to identify needs for tourism research as a research subject itself in Mexico. The study postulates five research needs for tourism studies in the country: broadening the number of topics related to tourism as a multidimensional entity; studying its research trends by adopting more encompassing methods; analyzing the current state of specific topics; identifying author's and journals' leaderships in the national context and; incorporating national knowledge to the international debates of tourism.

Keywords: Tourism research, tourism knowledge, trends, tourism journals, Mexico.

Fecha de recepción: 18 de mayo de 2015. Fecha de aceptación: 13 de enero de 2016.

CÓMO CITAR: Monterrubio, C. (2017). Necesidades de la investigación turística en México: reflexiones en el marco de la investigación internacional. Dimensiones Turísticas, 1(1), 31-52. https://doi.org/10.47557/YYHJ 9671 


\section{INTRODUCCIÓN}

$\mathrm{E}$ I turismo como campo de estudio ha ganado reconocimiento y un espacio relevante dentro de distintas comunidades académicas alrededor del mundo. Tal reconocimiento se ha manifestado en diferentes indicadores, pero de manera muy importante en los avances de la investigación turística internacional. Una parte muy considerable de ellos ha sido publicada y difundida en revistas especializadas (journals) en turismo en el mundo anglosajón; aunque de forma limitada, el estado que guardan estos avances puede ser un referente fundamental para analizar la investigación turística internacional y nacional específica. Sin embargo, la investigación turística en el mundo no se limita de ningún modo a la producción anglosajona; cualquier análisis de las tendencias o del estado de la investigación turística en el nivel global debe contemplar las particularidades de la producción académica en otros contextos, o al menos reconocer su existencia.

Analizar el estado que guarda la investigación turística internacional en cualquiera de sus indicadores tiene mucha relevancia. Por un lado, permite conocer los avances en cuanto a la construcción del conocimiento turístico desde perspectivas presuntamente globales, así como los vacíos existentes al respecto. Por otro lado, hace posible examinar cómo se ha construido ese conocimiento en términos conceptuales, teóricos, metodológicos, geográficos y empíricos, y quiénes han liderado tal construcción. Empero, debe tenerse presente que la investigación turística es una construcción heterogénea en su interior; su desarrollo ha sido diverso con relación a sus abordajes, incluyendo las construcciones específicas que cada país ha generado al respecto. Por tanto, el análisis de la investigación turística, a cualquier nivel, debe ser cuidadoso y reconocer su carácter multidimensional al existir diferentes factores que pueden indicar el estado que guarda la investigación.

Las reflexiones sobre la investigación turística en el ámbito nacional son también muy relevantes. Los análisis entre las tendencias de la investigación turística en el nivel internacional y las del nacional permiten comparar los avances en el contexto de países específicos con las tendencias posiblemente globales. Esto a su vez lleva a identificar oportunidades y necesidades de investigación turística tanto en el marco global como nacional. Debido a su importancia turística y sus implicaciones en los planos mundial, nacional, regional y local, México no debe estar exento del estudio de su investigación turística en el marco de la producción científica internacional.

En este contexto, el presente artículo tiene como propósito identificar los avances y necesidades de investigación en torno al estado que guarda la investigación turística como objeto de estudio en México, a partir del análisis de los avances reportados en la investigación turística internacional, con base en la revisión de publicaciones sobre el estado de la investigación turística internacional y nacional.

Para lograr el propósito planteado, este texto primero ofrece una serie de análisis y reflexiones en torno a indicadores que reflejan el estado de la investigación turística en el contexto anglosajón; posteriormente presenta algunas reflexiones en cuanto a avances y necesidades de investigación para el campo en el marco de la investigación turística en México. 


\section{Monterrubio}

\section{Marco metodológico}

La fundamentación principal de este trabajo recae en las reflexiones del autor a partir de lecturas focalizadas en el turismo como campo de estudio, las cuales fueron en gran medida de artículos obtenidos de revistas científicas internacionales especializadas en turismo, particularmente journals, y, en el caso de México, también de capítulos de libros, todos ellos enmarcados en el tema de la investigación turística.

Respecto a las revistas internacionales, la búsqueda se basó en trabajos en inglés debido a que estudios recientes revelan que poco más de $50 \%$ de las revistas en turismo en el mundo se publican en ese idioma (McKercher y Tung, 2015). De gran utilidad fueron las bases de datos de ScienceDirect y Google Scholar, de donde se extrajeron gran parte de los artículos, principalmente de las tres revistas con mayor reconocimiento en investigación turística en el mundo: Annals of Tourism Research (ATR), Tourism Management (TM) y Journal of Travel Research (JTR) (Gursoy y Sandstrom, 2016). Los criterios de búsqueda fueron "tourism research", "tourism knowledge" y "tourism journal assessment", en inglés, e "investigación turística” y "conocimiento turístico", en español.

Con excepción del trabajo requerido para el análisis de los avances en México, ninguna otra búsqueda se delimitó intencionalmente ni en tiempo ni espacio. Los criterios de búsqueda para ambas bases de datos se abrieron en estos dos aspectos con la intención de tener una base de fuentes bibliográficas de carácter longitudinal y obtener trabajos con análisis generales más que de contextos geográficos particulares.

\section{InVESTIGACIÓN TURíSTICA INTERNACIONAL}

La investigación turística internacional es relativamente reciente, en especial si se compara con el desarrollo de disciplinas o ciencias tradicionales. Debido a la importancia que el turismo ha tenido como actividad económica, sociocultural y medioambiental en gran parte del mundo, la investigación turística ha sido un ejercicio constante en varios países; además, ha experimentado cambios rápidos no solo en los tópicos de la investigación, sino también en sus abordajes conceptuales y metodológicos, y en su desarrollo teórico como tal en las últimas cuatro décadas (McKercher y Tung, 2015). Esto se evidencia ampliamente en el cuerpo de conocimientos y prácticas específicas en torno a la investigación turística en el nivel internacional. Si bien es prácticamente imposible analizar la investigación turística como un ente indiferenciable en su interior, su estado y tendencias pueden ser examinados a partir de los contenidos en revistas académicas anglosajonas. Teniendo en cuenta que algunas de estas revistas pueden considerarse representativas o barómetros de las tendencias temáticas en la investigación turística (Sharpley, 2011), sus contenidos, por tanto, son de alguna forma indicativos de los esfuerzos alrededor de la investigación internacional en el campo.

En el ámbito internacional, particular y notablemente en el marco de las publicaciones anglosajonas, las tendencias y el estado de la investigación turística han sido objeto 
de estudio académico. El proceso de búsqueda y análisis de este trabajo reveló que los estudios al respecto han intentando conocer el estado que guarda o ha guardado la investigación turística en distintas dimensiones y periodos concretos. Este proceso, basado en los criterios de búsqueda arriba descritos, permitió generar categorías formadas por la correspondencia o similitud de los objetivos o contenidos de los trabajos revisados, y se pudieron identificar las siguientes seis categorías principales:

- Tendencias temáticas generales

- Estados por tópicos específicos

- Tendencias metodológicas

- Tendencias nacionales

- Autores líderes

- Revistas líderes

Cada una de estas categorías es abordada, ilustrada y comentada a continuación.

\subsection{Tendencias temáticas generales en la investigación turística internacional}

Este tipo de estudios ha intentando identificar y analizar los tópicos abordados con mayor frecuencia dentro de la investigación turística en general. Los hallazgos reportados se han basado en el análisis del contenido de una o varias revistas (journals) especializadas en el campo del turismo. Bajo sus propios criterios, los investigadores en esta categoría han determinado los temas más recurrentes a partir de la selección de revistas reconocidas internacionalmente y en el marco de periodos específicos.

Con base en el análisis de una de las revistas ampliamente reconocidas en el nivel mundial, ATR, Xiao y Smith (2006b) ofrecen una lista de los tópicos más abordados en la investigación turística. Tomando en consideración los volúmenes publicados desde sus orígenes (1973) hasta 2003 de esta revista, los autores identificaron 27 áreas temáticas principales que demuestran una estabilidad notable en todo este periodo. Fueron 13 los temas que consistentemente aparecieron como tendencias de la investigación turística: metodología, desarrollo, impactos, organización y asociación, Estados Unidos, turista, turismo internacional, planeación, resort, cultura, mercadotecnia, motivación y atractivos. Las 14 áreas temáticas restantes, aunque sin mostrar consistencia en el transcurso de esos años, fueron: congresos, industria, destino, Tercer Mundo, empleo, turismo, hotel, política, demanda, transporte, turismo nacional, el Caribe, etnias, y gobierno.

Así, los autores concluyeron que la investigación turística se ha creado bajo dos metacategorías de áreas temáticas: por un lado, el área de metodología y constructos teóricos, y por otro, el desarrollo y los impactos (véase asimismo Xiao, Jafari, Cloke y Tribe, 2013), y también que "el crecimiento del conocimiento acerca de la metodología y los constructos teóricos es indicativo de los esfuerzos de un campo joven en alcanzar el rigor y ser reconocido como un campo ‘serio’ de investigación” (Xiao y Smith, 2006b, p. 503). 


\section{Monterrubio}

A partir del análisis de estudios de caso como metodología en el turismo, Xiao y Smith (2006a) ofrecen además una lista de tópicos que de alguna manera representan tendencias temáticas en la investigación turística, al menos en el marco, en esta ocasión, de cuatro revistas: ATR, JTR, TM y Tourism Analysis (TA), y durante el periodo 2000-2004. Los tópicos son el desarrollo turístico, la planeación, las percepciones de la comunidad y las reacciones a los impactos del turismo. Asimismo, los autores mostraron que temas como formas alternativas de la experiencia turística, desarrollo profesional, marketing o imagen de los destinos, segmentación o mercados turísticos, turismo cultural, relaciones residentes-turistas, así como operaciones y gestión de la industria, son también temas abordados repetidamente en la investigación publicada en inglés. Cabe mencionar que, de acuerdo con el mismo estudio, estos temas son tratados principalmente por académicos adscritos a universidades o institutos de investigación pertenecientes a las áreas de turismo, hospitalidad y recreación, y a los llamados países desarrollados.

De manera similar, Ballantyne, Packer y Axelsen (2009) revelaron que los temas abordados en el turismo han sido de alguna forma constantes durante los últimos años. Para ello revisaron 2868 artículos publicados de 1994 a 2004 en 12 journals de alto reconocimiento internacional. De acuerdo con los autores, las cuatro áreas temáticas más frecuentemente tratadas en la investigación turística han sido: turista/visitante, en particular comportamientos, preferencias y perspectivas; destinos, incluyendo imagen, gestión y desarrollo; planeación turística en su dimensión de desarrollo turístico, estrategias, predicciones y proyecciones; y mercadotecnia, abarcando marketing, segmentación y promoción. Según el estudio, estos temas representan 37 \% del total de la producción científica en la investigación turística. En el trabajo de Sharpley (2011) se encontraron tendencias muy similares, pero con base en el análisis de la producción científica de 2005 a 2010 en ATR, TM, International Journal of Tourism Research (IJTR) y Current Issues in Tourism (CIT).

Por su parte, el estudio de Tribe y Xiao (2011), a partir del examen de las publicaciones también en ATR en el periodo 2010-2011, indicó que, en el contexto de las ciencias sociales, los temas más abordados incluyen: reflexiones sobre los estudios turísticos y su producción del conocimiento; desarrollo, impactos y comunidades; y autenticidad y experiencia turística.

El cuadro 1 permite comparar los hallazgos de los estudios en términos de los tópicos más abordados en la investigación turística anglosajona. Si bien cada estudio persiguió de alguna forma conocer las tendencias en la investigación turística internacional, es claramente observable que los resultados al respecto no han sido consistentes, por lo que no se sabe con precisión cuáles son esas tendencias ni el estado que guarda la investigación en torno a los temas generales tratados.

Los estudios antes mencionados, y algunos otros (como Swain, Brent y Long, 1998), sugieren un panorama de los temas más abordados dentro de la investigación turística internacional; sin embargo, estos hallazgos deben tomarse con cierta precaución, ya que, por ejemplo, esos estudios se han basado en un número muy específico de revistas científicas y dentro de periodos definidos. Aunque las revistas comúnmente consideradas 
CuADro 1. Tendencias temáticas de la investigación turística en la bibliografía anglosajona Cuadro comparativo (lista seleccionada)

\begin{tabular}{|c|c|c|c|c|c|c|}
\hline Estudio & $\begin{array}{c}\text { Swain, Brent y } \\
\text { Long (1998) }\end{array}$ & $\begin{array}{l}\text { Xiao y Smith } \\
\text { (2006b) }\end{array}$ & $\begin{array}{l}\text { Ballantyne et al. } \\
\text { (2009) }\end{array}$ & $\begin{array}{c}\text { Xiao y Smith } \\
\text { (2006a) }\end{array}$ & $\begin{array}{l}\text { Tribe y Xiao } \\
\text { (2011) }\end{array}$ & $\begin{array}{l}\text { Sharpley } \\
\text { (2011) }\end{array}$ \\
\hline Fuentes & ATR & ATR & 12 journals & $\begin{array}{l}\text { Estudios de } \\
\text { caso ATR, } \\
\text { JTR, TA y TM }\end{array}$ & ATR & $\begin{array}{l}\text { ATR, TM, CIT } \\
\text { e IJTR }\end{array}$ \\
\hline Periodo & $\begin{array}{l}\text { Primeros } 25 \\
\text { volúmenes }\end{array}$ & $1973-2003$ & 1994-2004 & $2000-2004$ & $2009-2010$ & $2005-2010$ \\
\hline \multirow{10}{*}{$\begin{array}{l}\text { Temas } \\
\text { principales }\end{array}$} & Impactos & Metodología & Turista/visitante & Desarrollo & $\begin{array}{l}\text { Los estudios } \\
\text { turísticos y la } \\
\text { producción del } \\
\text { conocimiento }\end{array}$ & $\begin{array}{l}\text { Turista/ } \\
\text { visitante }\end{array}$ \\
\hline & Organizaciones & Desarrollo & Destinos & Planeación & $\begin{array}{l}\text { Desarrollo, } \\
\text { impactos y } \\
\text { comunidades }\end{array}$ & Marketing \\
\hline & Desarrollo & Impactos & $\begin{array}{l}\text { Planeación } \\
\text { turística }\end{array}$ & $\begin{array}{l}\text { Percepciones } \\
\text { comunitarias } \\
\text { e impactos } \\
\text { del turismo }\end{array}$ & $\begin{array}{l}\text { Autenticidad } \\
\text { y experiencia } \\
\text { turística }\end{array}$ & Destinos \\
\hline & Métodos & $\begin{array}{l}\text { Empresa y } \\
\text { asociación }\end{array}$ & Marketing & & $\begin{array}{l}\text { Tipologías de los } \\
\text { turistas }\end{array}$ & Gestión \\
\hline & & $\begin{array}{l}\text { Estados } \\
\text { Unidos }\end{array}$ & Turismo cultural & & $\begin{array}{l}\text { Destinos y } \\
\text { atractivos }\end{array}$ & $\begin{array}{l}\text { Aspectos } \\
\text { económicos }\end{array}$ \\
\hline & & Turista & $\begin{array}{l}\text { Aspectos } \\
\text { económicos }\end{array}$ & & $\begin{array}{l}\text { Motivación } \\
\text { turística, } \\
\text { comportamiento } \\
\text { y toma de } \\
\text { decisiones }\end{array}$ & Métodos \\
\hline & & $\begin{array}{l}\text { Turismo } \\
\text { internacional }\end{array}$ & Impactos & & $\begin{array}{l}\text { Cultura y } \\
\text { patrimonio }\end{array}$ & $\begin{array}{l}\text { Turismo } \\
\text { cultural }\end{array}$ \\
\hline & & Planeación & $\begin{array}{l}\text { Tendencias del } \\
\text { turismo }\end{array}$ & & $\begin{array}{l}\text { Representación, } \\
\text { identidad e } \\
\text { imagen }\end{array}$ & Planeación \\
\hline & & Resort & $\begin{array}{l}\text { Investigación y } \\
\text { métodos }\end{array}$ & & & $\begin{array}{l}\text { Comunidad, } \\
\text { anfitriones e } \\
\text { invitados }\end{array}$ \\
\hline & & Cultura & Hospitalidad & & & Política \\
\hline
\end{tabular}

Fuente: Elaboración propia.

han sido calificadas como representativas de las tendencias de la investigación turística (Sharpley, 2011), han quedado excluidos por completo los contenidos de la gran mayoría de revistas que pudieran existir en el mundo, no solo las especializadas en turismo sino en muchas otras áreas del conocimiento en donde la investigación en torno al turismo ha sido también publicada. Si se tiene en cuenta que las tendencias varían según la revista seleccionada, como lo revelan algunos estudios (Xiao y Smith, 2006a), las tendencias de la investigación turística reportadas dependerán en gran medida de las fuentes de análisis y de 


\section{Monterrubio}

los indicadores específicos que el investigador haya seleccionado. Este tipo de estudios ha excluido otras fuentes importantes que concentran también tendencias de la investigación turística: libros, memorias de congresos, proyectos de investigación, y tesis de doctorado y maestría, y en algunos países también las de nivel licenciatura, no han formado parte del análisis de tales tendencias. Se observa asimismo que los procesos metodológicos de los estudios han sido distintos, lo cual inevitablemente tendrá repercusiones al intentar cualquier homogeneización de sus hallazgos.

Así pues, la generalización y representatividad de los resultados acerca de las tendencias temáticas de la investigación en turismo son muy limitadas. Por esta razón, los resultados hasta ahora obtenidos, si bien valiosos e indicativos, son únicamente una representación parcial, seleccionada y muy bien demarcada en sus alcances, de las tendencias de investigación turística en el nivel internacional.

\subsection{Estados por tópicos específicos en la investigación turística internacional}

En esta categoría se encuentran los estudios que han perseguido conocer los avances realizados así como los vacíos dentro de un tópico específico del turismo. Dado que el turismo es un fenómeno con múltiples dimensiones económicas, socioculturales, políticas, medioambientales, empresariales, entre muchas otras, no sorprende que varios aspectos de ellas hayan sido abordados académicamente y tratados como temas particulares de análisis; así pues, los estudios en esta categoría han analizado las tendencias específicas y los avances que experimentan algunos de estos temas dentro de la investigación turística. Sin embargo, se observa que el abordaje de estos no ha sido uniforme: mientras que existen tópicos muy concretos del turismo que han sido revisados repetidamente, existen muchos otros que permanecen poco o nulamente explorados. Esto evidencia que la investigación turística, en su interior, no se ha desarrollado de igual manera, y por lo tanto complica reflejar su estado como un todo.

La dificultad en querer establecer tendencias de la investigación global o el estado que esta guarda puede observarse en la comparación de la evolución temática específica del mismo. Algunos casos, seleccionados a juicio del autor, pueden ilustrar este punto. Los avances en el emprendedurismo en el turismo, por ejemplo, han sido estudiados en su particularidad; al respecto se ha reportado que el trabajo empírico acerca de este tema no ha incrementado durante las últimas dos décadas, y que el trabajo teórico en el mismo es aún limitado, existiendo también una falta de sofisticación metodológica (Li, 2008). De manera similar, los estudios sobre el género en el turismo, aunque experimentan un crecimiento, mantienen todavía un estado marginado dentro de los estudios turísticos; sus tendencias internacionales se concentran básicamente en cuatro subtemas: el consumo, los impactos en comunidades locales, el trabajo, y la teoría misma (Figueroa-Domecq, Pritchard, SegoviaPérez, Morgan y Villacé-Molinero, 2015). 
A diferencia de las dos anteriores, la investigación sobre el turismo deportivo parece mostrar un mayor avance; el turismo deportivo como tópico de investigación parece estar alcanzando una etapa de madurez reflejada en una conceptualización fuerte, la fundamentación de trabajo empírico por teorías adecuadas, la aplicación transparente y robusta de métodos, una comunidad clara de académicos con amplio interés en el área, y una revista especializada, The Journal of Sport \& Tourism (Weed, 2009); sus dos tendencias principales se presentan en el comportamiento, perfiles y motivaciones de los turistas deportivos, por un lado, y los impactos, por otro (Weed, 2006).

En la misma línea, se encuentra el caso de las investigaciones económicas en el turismo. Aunque la economía del turismo ha contribuido tradicionalmente al amplio campo del turismo, en el nivel macro llevará aún mucho tiempo para que este tema de investigación alcance una madurez metodológica, además de que exige una fundamentación teórica mayor; las tendencias en su estudio se concentran ampliamente en la demanda, la oferta, el impacto macroeconómico y aspectos medioambientales (Song, Dwyer, Li y Cao, 2012).

En cuanto a los impactos del turismo, otro tópico dentro de la investigación turística global, el desarrollo en su investigación ha permitido identificar los cambios percibidos por los residentes locales, pero no ha podido explicar el porqué de tales percepciones; la investigación existente sobre los impactos del turismo revela que hay cuatro etapas clave en el desarrollo de su investigación que abordan los debates sobre las definiciones, marcos conceptuales, desarrollo de instrumentos y prueba de los mismos (Deery, Jago y Fredline, 2012); la investigación especializada en este tema, sin embargo, se ha realizado con una gran carencia teórica (Sharpley, 2014).

El estado que guardan estos y otros tópicos como turismo urbano (Ashworth y Page, 2011), empleo en el turismo (Johnson y Thomas, 1990), motivaciones turísticas (Dann, 1981), imagen del destino (Pike, 2002), entre muchos otros, al menos en la literatura anglosajona, señala que el abordaje y el desarrollo de los temas específicos en la investigación turística global han sido desiguales. Mientras que algunos temas parecen estar alcanzado un avance conceptual y teórico, otros son aún muy incipientes en su abordaje, lo cual, estrictamente hablando, imposibilita analizar el estado del conocimiento turístico en general, pues no solo debería tenerse conocimiento preciso sobre todos los temas que se han abordado en su particularidad, sino también una evaluación cuantitativa y cualitativa, bajo parámetros comparables, del avance en cada uno de ellos.

\subsection{Tendencias metodológicas en la investigación turística internacional}

En el análisis de las tendencias del turismo también se han abordado los usos metodológicos en el campo. Los estudios relacionados han perseguido conocer los métodos y técnicas específicos más utilizados dentro de los estudios turísticos. Aunque son muy pocos los trabajos que se han concentrado en examinar el estado metodológico de tales investigaciones en el mundo anglosajón (menos aún en otros idiomas), los métodos de 


\section{Monterrubio}

investigación en turismo per se han sido objeto de análisis. Estos estudios se han enfocado, por ejemplo, en la investigación cuantitativa versus cualitativa (Walle, 1997), el estado de la investigación cualitativa (Riley y Love, 2000), las tendencias metodológicas de investigación (Ballantyne, Packer y Axelsen, 2009), los estudios de caso (Xiao y Smith, 2006a) y la investigación conceptual (Xin, Tribe y Chambers, 2013), todos ellos en el ámbito del turismo.

En cuanto a las tendencias metodológicas, los estudios han revelado que existe un dominio amplio de métodos cuantitativos en la investigación turística, el cual, al menos en un principio, podría explicarse en la sofisticación numérica y estadística necesaria para el progreso de la investigación en el campo (Riley y Love, 2000; Walle, 1997). Sin embargo, la investigación turística ha reconocido la legitimidad de diversas metodologías para su abordaje, entre ellas las de corte cualitativo, que más allá de contradecir a las cuantitativas, las complementan (Decrop, 1999; Walle, 1997), y las de corte conceptual (Xin et al., 2013) que, aunque son minoría, poseen relevancia en el desarrollo del campo. De esta forma, los estudios con enfoques sociológicos y antropológicos principalmente han rescatado el valor de los métodos cualitativos en el turismo, llevando a estos a una creciente popularidad (Phillimore y Goodson, 2004).

Hoy en día no es sorprendente que tanto los métodos cuantitativos como cualitativos y conceptuales formen parte de la investigación turística internacional. Aunque obtener datos representativos del verdadero estado metodológico que guarda la investigación en el campo en el nivel global constituye prácticamente una imposibilidad, algunos estudios sugieren que los métodos cuantitativos en la investigación turística siguen dominando y los conceptuales permanecen como minoría. Según Ballantyne et al. (2009), de 1994 a 2004 la mayoría de los estudios realizados en turismo (59 \%) ha adoptado diseños cuantitativos, otra parte (19\%) ha utilizado métodos cualitativos y la minoría (6\%) ha recurrido a métodos mixtos; el resto (16 \%) han sido trabajos de revisión o netamente teóricos sin una metodología de este tipo. Cifras similares han sido ofrecidas por investigaciones previas con el mismo objeto de estudio (Xiao y Smith, 2006a).

\subsection{Tendencias nacionales en la investigación turística internacional}

Dentro del turismo, se debe tener presente que la investigación ha experimentado un desarrollo distinto en diversos entornos geográficos del mundo. En la bibliografía especializada, existen también trabajos de investigación que han buscado conocer los avances realizados y, en algunos casos, las tendencias en términos de investigación turística en el contexto de países o regiones específicos. Los resultados han sido variados, cada país con temas y abordajes particulares.

En el caso de Argentina, por ejemplo, la investigación en el campo se ha centrado sobre todo en cuestiones como recursos turísticos, turismo y medio ambiente, oferta y demanda (Castellucci, 2001), mientras que en Grecia, la producción científica se ha concentrado 
ampliamente en la planeación y las dimensiones económicas (Galani-Moutafi, 2004). En otros países, como España, algunos de los tópicos más estudiados son: destinos, gestión, desarrollo, economía, mercados, comportamiento del turista, hoteles y satisfacción (Albacete-Sáez y Fuentes-Fuentes, 2010). En China, los temas más abordados han sido turista/ visitante y aspectos económicos, que juntos componen 25 \% de la producción nacional; cabe mencionar que en ese país mucha de la investigación en turismo es aplicada u orientada a la práctica en un contexto local (Bao, Chen y Ma, 2014), lo cual representa una dificultad pero a la vez una oportunidad para su integración al análisis del estado de la investigación turística internacional.

Según los casos de los países antes citados y de otras naciones y regiones como Australia (Bushell, Prosser, Faulkner y Jafari, 2001), México (Osorio, Castillo y Moreno, 2007) o Latinoamérica (Schlüter y Bertoncello, 2010), por señalar algunos, los temas específicos en cada uno son distintos y tratados desde perspectivas diferentes, muchas veces obedeciendo a diversos factores propios del país o región en cuestión. El análisis de las tendencias de la investigación no puede basarse únicamente en indicadores cuantitativos sino también cualitativos; es decir, además de diferenciar por el número y orden de temas abordados por país, debe examinarse el avance, utilidad, impacto y contribución que en cada uno de ellos se ha tenido en el contexto del conocimiento de la nación respectiva.

\subsection{Autores líderes en la investigación turística internacional}

Los estudios en el mundo anglosajón han buscado también establecer listas (rankings) y posiciones de los autores en la investigación turística, y con ello conocer su liderazgo, según sus aportaciones al campo. Estudios de este tipo han fundamentado sus análisis, como en el caso de los temas o métodos, en las contribuciones de revistas específicas, seleccionadas con criterios particulares de cada investigador.

El trabajo de Sheldon (1991) es uno de los pioneros al respecto. Basada en la producción de la década de 1980 de tres journals, ATR, JTR y TM, la autora estadounidense reveló que los investigadores de Estados Unidos y Canadá (65\%), seguidos por los europeos (24.8\%), representan la proporción más grande de las contribuciones. Los países con mayor aportación a la investigación turística fueron en ese entonces Estados Unidos, Reino Unido y Canadá; la autora concluyó que los investigadores de estas naciones, más que de otras regiones del mundo, son quienes producen y dan forma al conocimiento turístico. Es de interés mencionar también que, a más de tres décadas del estudio de Sheldon, la contribución a los estudios turísticos en revistas principales, particularmente en ATR, sigue dominada por académicos de países anglosajones, en concreto de Estados Unidos, Reino Unido, Canadá, Australia y Nueva Zelanda (Xiao et al., 2013).

Otros trabajos, como el de Ryan (2005), han sido mucho más específicos y, con base en la cantidad de sus contribuciones en estas revistas, han enlistado, desde su perspectiva, los nombres de los autores líderes en la investigación turística, como John L. Crompton, 


\section{Monterrubio}

Chris Ryan y Joseph O'Leary, con un total de 92, 58 y 53 publicaciones en esas revistas, respectivamente, de 1990 a 2004.

La obra de Zhao y Ritchie (2007) sobre el liderazgo académico en la investigación turística, que también parte de la cantidad de artículos publicados de 1985 a 2004 en ocho journals, ofrece datos interesantes. Los autores identificaron a los 57 académicos más prolíficos a partir de publicar al menos 11 artículos en el periodo. Daniel Fesenmaier, Chris Ryan y John Crompton aparecen como los tres académicos líderes. Algunos datos merecen observación aparte. Por ejemplo que, excepto por cuatro de los investigadores, todos obtuvieron su grado doctoral en universidades de países anglosajones, y la gran mayoría también estaba adscrita a universidades del mismo tipo. Adicionalmente se puede advertir que, en el marco del estudio, existen al menos dos grupos subrepresentados, uno basado en la nacionalidad y el otro en el género. Al respecto, el estudio señaló que ningún académico latinoamericano se encuentra en la lista y que hay un dominio muy pronunciado del género masculino al existir solo seis mujeres, al menos dentro de los 57 primeros lugares, en el liderazgo de la investigación turística anglosajona.

Determinar el liderazgo académico a partir de la cantidad de publicaciones es por supuesto cuestionable. Esto se puede evidenciar en que estudios similares, pero con base en el análisis de las citas de los trabajos y no en la cantidad de publicaciones, han revelado listas distintas en torno a cuántos, quiénes y en qué posición las conforman (McKercher, 2008; véase también McKercher y Tung, 2015). Si se piensa que el impacto y la calidad podrían jugar un papel más importante en comparación con la cantidad, los resultados serían con seguridad diferentes. Otros aspectos relevantes al respecto podrían ser, también debatiblemente, el índice de citación de los trabajos, tanto de artículos en revistas científicas como de libros, y la trascendencia conceptual, teórica y práctica de los mismos. Considerando que el turismo es multitemático, sería quizás más recomendable precisar el liderazgo cuantitativa y cualitativamente en función de temas o áreas geográficas específicos de la investigación turística, y no tanto de la investigación turística en general.

\subsection{Revistas líderes en la investigación turística internacional}

En el análisis de los avances de la investigación turística internacional se encuentra también el interés en conocer el ranking de las revistas académicas especializadas en turismo. Las revistas académicas son útiles en la producción, diseminación e intercambio del conocimiento; en la evaluación de la investigación; y en la identificación del estado académico de los investigadores, departamentos e instituciones (Hall, 2011). Igual que en muchas otras áreas del conocimiento, estas revistas han cumplido un rol fundamental en reconocer al turismo como campo de estudio y área de investigación científica, pues son consideradas la principal plataforma de la investigación en un campo de conocimiento determinado (Cheng, Li, Petrick y O’Leary, 2011). 
Muchas de las revistas académicas en turismo son de reciente creación. Aunque la revista especializada más antigua, Tourism Review, data de 1946, el crecimiento de estas publicaciones se hace muy presente desde la década de 1990, no solo en cantidad sino además en la diversidad de temas dentro del turismo. En los noventa fue tal el surgimiento de nuevas revistas que este periodo ha sido llamado "the explosion of new journals" (Goeldner, 2005). El crecimiento de las revistas en distintos idiomas ha sido significativo en las últimas décadas. Mientras que para inicios de los noventa se estimaba que existían menos de 30 revistas en turismo y hospitalidad en el idioma inglés, y para 2006 se contabilizaban 70 e incluso 100 revistas en el área (McKercher, Law y Lam, 2006), para 2011 el número se estimaba en al menos 150 (Cheng et al., 2011). Es importante notar, sin embargo, que estas cifras con frecuencia registran únicamente aquellas revistas con cierto reconocimiento y/o popularidad en el idioma inglés.

No obstante, el número de revistas especializadas en el área resulta mucho mayor si se tienen en cuenta aquellas que son publicadas en otros idiomas y en distintas partes del mundo. Quizás uno de los esfuerzos más valiosos en realizar recientemente un listado muy extenso, aunque no exhaustivo, de las revistas enfocadas al turismo en diversos idiomas en el mundo es el efectuado por McKercher (2013). De acuerdo con su registro, a septiembre de 2013 se contabilizaban 250 revistas especializadas en turismo y en aspectos muy cercanamente relacionados como la hospitalidad y los eventos; al 2014, la cifra se calculaba en 290, de las cuales 150 publican en inglés (McKercher y Tung, 2015). Esta cifra, sin embargo, ya es obsoleta puesto que el número actual es mayor debido a que desde esa fecha se han incorporado revistas nuevas a la lista internacional, entre ellas Journal of Tourism Futures, en Holanda (2014), Tourism Dimensions, en India (2014), Journal of Tourism Cities, en Reino Unido (2015), y Dimensiones Turísticas, en México (2017).

Más que en la cantidad de revistas, el interés académico principal se ha concentrado en la posición que estas ocupan, según la calidad de sus trabajos. Se trata de ejercicios de investigación realizados sobre todo desde la década de 1990. Al respecto existen varios trabajos que, particularmente bajo dos metodologías, encuestas a expertos y reportes de citación, han enlistado las revistas con mayor calidad e impacto. Los resultados han variado en su composición y muestras de estudio, pero, a diferencia de la categoría de los temas más abordados, son muy consistentes en cuanto a las revistas que ocupan los primeros lugares, esto también por supuesto en el contexto anglosajón.

De este modo, el trabajo de Sheldon (1990), basado en una encuesta a 103 investigadores pertenecientes a asociaciones del campo del turismo y la hospitalidad en 1989, reportó que ATR, JTR y TM eran percibidas como las revistas de mayor calidad en ese entonces. Con base también en una encuesta a 142 miembros de dos asociaciones internacionales, y reconociendo que el perfil geográfico de los investigadores influye en el ranking percibido, Pechlaner, Zehrer, Matzler y Abfalter (2004) revelaron que, por parte de miembros fuera de Estados Unidos, las revistas con mayor posicionamiento fueron las tres encontradas por Sheldon, aunque en orden distinto: ATR, TM y JTR. Los trabajos de Ryan (2005), McKercher, Law y Lam (2006), y muy recientemente Gursoy y Sandstrom (2016), 


\section{Monterrubio}

confirman que, en el mundo anglosajón, ATR, TM y JTR son percibidas y reportadas como las revistas académicas en turismo de mayor calidad.

Como se deduce en las secciones anteriores, la investigación turística entre las naciones se ha desarrollado de manera desigual, bajo distintos tópicos -generales y específicos-, abordajes, enfoques y avances, lo cual exige revisar de modo más crítico la supuesta madurez de la misma (Zhao y Ritchie, 2007; Schlüter y Bertoncello, 2010) y el estado del conocimiento del turismo. El hecho de que la investigación turística en algunos países se encuentre aún en etapas incipientes no ha sido incorporado en las afirmaciones globales de la supuesta madurez y el análisis de las tendencias y estado del conocimiento del campo como una actividad mundial. De esta forma, la investigación turística muy probablemente no ha alcanzado una madurez en otras partes del mundo y bajo otras prácticas académicas, parcial o totalmente ajenas a los cánones del mundo anglosajón, sobre todo en los llamados países en vías de desarrollo en donde tradicionalmente el avance de la ciencia en general ha sido más limitado y los componentes de la investigación turística no han obedecido necesariamente a las posibles tendencias internacionales.

\section{Investigación turística en México}

La investigación turística en México, en sus antecedentes más importantes, se remonta a los años sesenta, y su desarrollo ha pasado por enfoques netamente económicos, sociales, políticos y medioambientales, aunque su reto ha sido precisamente la comprensión del turismo desde una perspectiva integral (Jiménez y Sosa, 2006). Los trabajos del Instituto Mexicano de Investigación Turística (IMIT) pueden indicarse como los antecedentes más relevantes; estos se enfocaron en la obtención de datos para efectos prácticos y con particular orientación hacia la planeación, promoción y comportamiento de la demanda. Las investigaciones del Centro de Estudios Superiores del Turismo (Cestur) (actualmente Instituto de Competitividad Turística, Ictur), dependiente de la Secretaría de Turismo del país, amplían la variedad de temas abordados a partir de 1980, incorporando, además de los estudios para la inversión y planeación, cuestiones como perfil de los turistas, centros de afluencia de visitantes, y factibilidad del turismo cultural (Castillo y Lozano, 2006; Guevara, Molina y Tresserras, 2006).

De esta manera, como observa Hiernaux (2000), en muchas de las investigaciones en el campo en México han prevalecido las dimensiones administrativas del turismo. La consulta de algunos eventos académicos recientes demuestra esta situación en la actualidad. Por ejemplo, el Congreso de Investigación Turística Aplicada de la Red de Investigadores y Centro de Investigación en Turismo (RICIT), integrada por investigadores y académicos destacados por su producción científica en el campo, en su edición 2015 definió cinco temáticas en el marco de la innovación en el turismo: formación profesional, gestión de destinos, desarrollo de productos, organizaciones turísticas, y tendencias gastronómicas (www.ricit. sectur.gob.mx), las cuales manifiestan la orientación que tienen muchas investigaciones 
en el país. Esto ha sido quizá como consecuencia, por un lado, de los intereses particulares de las instituciones federales, pero por otro lado, la investigación turística en México ha respondido también al perfil de las universidades e intereses de los investigadores, parte de lo que Hall (2011) denomina el mesonivel de la investigación turística. Así, la investigación académica en el campo no se ha ocupado necesariamente de los requerimientos del sector productivo, ni de las implicaciones sociales y ambientales del fenómeno (Espinosa, 2007, p. 18), ni de muchos otros alcances del turismo que han sido reconocidos en el nivel internacional. Por lo tanto, ampliar la diversidad de temas asociados a la complejidad que el turismo posee, tanto en su práctica en México como en su teoría, representa una primera necesidad de investigación para la comunidad científica del país.

Igual que en la investigación turística anglosajona, en México existen algunos trabajos -aunque en mucha menor medida- que a partir de diferentes fuentes de análisis han intentado identificar los temas más recurrentes en la agenda de investigación turística nacional. Con base en la revisión de algunas investigaciones en el país de los últimos años (2001-2005), Espinosa (2007), por ejemplo, ofreció un panorama sobre las tendencias de investigación en el campo. Al respecto, la autora encontró siete temáticas sobresalientes; las dos más destacadas fueron, por una parte, el análisis de los espacios y planeación de proyectos turísticos (22\%) y, por otra, el cuidado y conservación del ambiente, ecoturismo y sustentabilidad (18\%). Los temas restantes fueron currículum y capacitación; estado de la investigación turística; dirección y administración de empresas turísticas; políticas, reglamentación y normatividad en torno al turismo; e investigaciones de diversa vinculación social.

Al reconocer que existe dificultad para acordar criterios aplicables para el análisis de la caracterización de la RICIT, Jiménez, Osorio y Avila (2006) hacen una revisión de los proyectos registrados entre los 137 miembros de la Red identificando cinco temáticas principales: el turista y su contexto, la industria turística y su funcionamiento, la comunidad local, educación turística y epistemología, y conceptualización e investigación. Los autores reconocen, sin embargo, que esta clasificación es únicamente para fines del análisis perseguido y solo de carácter preliminar.

Por su parte, la Universidad Nacional Autónoma de México (Cestur, 2007) genera sus propias conclusiones en torno a las líneas de investigación turística que al 2007 se trabajaban en el país. El estudio concluyó que existen ocho grandes líneas temáticas (aunque también enlista los diez temas específicos) dentro de la investigación en el área; los dos principales grupos son: espacio, planeación, sustentabilidad y desarrollo local; y mercados, segmentos turísticos y desarrollo de nuevos productos. Los otros temas que se han abordado son: educación, capacitación y mercado de trabajo; estudios económicos y administrativos; turismo y cultura, patrimonio e identidad; sociedad y turismo; legislación, gestión y políticas públicas en turismo; y propuestas teórico-conceptuales.

Una revisión preliminar de los contenidos de fuentes primarias, específicamente de eventos académicos, arroja que los temas en turismo abordados en México en la actualidad no han variado mucho. El congreso anual de la Academia Mexicana de Investigación 


\section{Monterrubio}

Turística, A. C. (АМIT) (www.amiturismo.org), la asociación académico-científica de turismo más importante en el país, puede fungir como un referente para conocer los temas actuales. De acuerdo con las convocatorias de su congreso en los últimos cinco años, los contenidos de las ponencias han sido clasificados en temas mayores como planificación y desarrollo; administración y promoción; mercadotecnia; sustentabilidad; causas y efectos del turismo; y cultura, conocimiento, educación y tecnología.

Si bien revisiones como estas y otros estudios (véase también Jiménez, Osorio y Avila, 2006; Guevara et al., 2006; Osorio et al., 2007) ofrecen un panorama de los temas abordados en la investigación turística en México, sus hallazgos han sido basados en indicadores muy específicos de los trabajos, incluido el análisis de los congresos nacionales (Espinosa, 2007), los proyectos registrados en instituciones (Osorio et al., 2007), o las líneas de grupos específicos de investigación (Tamayo y Peñaloza, 2005).

Por tal razón, estos hallazgos deben tomarse con la debida precaución, pues al basarse en un solo indicador (o unos cuantos), brindan panoramas, aunque valiosos e indicativos, únicamente parciales de las tendencias temáticas de la investigación turística en México (véase cuadro 2). Es importante apuntar que, en una comparación, los temas tratados dentro de la investigación turística no son fácilmente identificables, pues se nota inconsistencia entre los resultados hasta ahora reportados.

Asimismo, es interesante observar que, en el caso de México, hay muy pocos trabajos, si no es que ninguno aún, que ofrezca un examen de esas tendencias con base en análisis bibliométricos quizás más indicativos. En concreto se advierte que el análisis de las publicaciones existentes en revistas especializadas (y no especializadas) para conocer el estado de la investigación turística, como han hecho otras comunidades académicas en el mundo, no ha sido prácticamente utilizado como un medio para conocer el estado o las tendencias de investigación turística en México, ya sea en su dimensión conceptual, teórica o metodológica. Esto representa otra necesidad de investigación para la comunidad científica nacional. Si se desea tener una comprensión más detallada y precisa sobre los avances en la construcción del conocimiento turístico en el país, es menester realizar estudios bibliométricos que a partir de fuentes primarias significativas -revistas científicas primordialmente- permitan ubicar el verdadero estado temático de la investigación producida en México.

Otra necesidad de investigación en México recaería no solo sobre la cantidad de temas que se han abordado, sino de las especificaciones de los abordajes. En el contexto de la investigación turística en México queda mucho por hacer en cuanto a la relevancia que tales abordajes han tenido en el desarrollo del conocimiento turístico nacional; analizar las evoluciones conceptuales, teóricas, metodológicas y la utilidad práctica de los temas que se han estudiado en el país permanece como una necesidad latente de investigación científica en el campo.

En el mismo sentido, el volumen de trabajos sobre los avances que se han hecho en torno a tópicos específicos del turismo en México es significativamente limitado, si no es que inexistente aún. Como se revela al inicio de este artículo, en un contexto internacional 
Cuadro 2. Tendencias temáticas de la investigación turística en México Cuadro comparativo

\begin{tabular}{|c|c|c|c|c|c|}
\hline Estudio & $\begin{array}{c}\text { Espinosa } \\
\text { (2007) }\end{array}$ & Osorio et al. (2007) & $\begin{array}{l}\text { Jiménez et al. } \\
(2006)\end{array}$ & Cestur (2007) & $\begin{array}{l}\text { Guevara et al. } \\
(2006)\end{array}$ \\
\hline Fuentes & $\begin{array}{l}\text { Congresos } \\
\text { nacionales }\end{array}$ & $\begin{array}{l}\text { Congresos } \\
\text { nacionales en } \\
\text { coordinación de IES } \\
\text { y Sectur }\end{array}$ & $\begin{array}{l}\text { Proyectos de } \\
\text { investigación e } \\
\text { investigadores Red } \\
\text { de Investigadores } \\
\text { y Centro de } \\
\text { Investigación en } \\
\text { Turismo }\end{array}$ & $\begin{array}{l}\text { Instituciones } \\
\text { de gobierno } \\
\text { y educación, } \\
\text { y congresos } \\
\text { nacionales }\end{array}$ & $\begin{array}{l}\text { Líneas de } \\
\text { investigación, } \\
\text { contenidos y } \\
\text { temas en Guevara } \\
\text { (2006) }\end{array}$ \\
\hline Periodo & 2001-2005 & 2004-2007 & 2006 & 2004-2007 & 2006 \\
\hline \multirow{10}{*}{$\begin{array}{l}\text { Temas } \\
\text { principales }\end{array}$} & $\begin{array}{l}\text { Espacios y } \\
\text { planeación }\end{array}$ & $\begin{array}{l}\text { Espacio, } \\
\text { planeación, } \\
\text { sustentabilidad, } \\
\text { desarrollo local }\end{array}$ & $\begin{array}{l}\text { Recursos y } \\
\text { atractivos }\end{array}$ & $\begin{array}{l}\text { Ordenamientos, } \\
\text { planeación y } \\
\text { espacio }\end{array}$ & $\begin{array}{l}\text { Gestión y políticas } \\
\text { públicas }\end{array}$ \\
\hline & $\begin{array}{l}\text { Conservación, } \\
\text { ecoturismo y } \\
\text { sustentabilidad }\end{array}$ & $\begin{array}{l}\text { Mercados, } \\
\text { segmentos, nuevos } \\
\text { productos }\end{array}$ & Educación turística & $\begin{array}{l}\text { Oferta y } \\
\text { productos } \\
\text { turísticos }\end{array}$ & $\begin{array}{l}\text { Planeación, } \\
\text { desarrollo y } \\
\text { sustentabilidad }\end{array}$ \\
\hline & $\begin{array}{l}\text { Currículo y } \\
\text { capacitación }\end{array}$ & $\begin{array}{l}\text { Educación, } \\
\text { capacitación y } \\
\text { trabajo }\end{array}$ & $\begin{array}{l}\text { Sustentabilidad e } \\
\text { impactos }\end{array}$ & $\begin{array}{l}\text { Comunidades } \\
\text { locales }\end{array}$ & $\begin{array}{l}\text { Educación, } \\
\text { capacitación, } \\
\text { trabajo y } \\
\text { tecnología }\end{array}$ \\
\hline & $\begin{array}{l}\text { Estado de la } \\
\text { investigación }\end{array}$ & $\begin{array}{l}\text { Economía y } \\
\text { administración }\end{array}$ & $\begin{array}{l}\text { Administración y } \\
\text { operación }\end{array}$ & Sustentabilidad & $\begin{array}{l}\text { Economía y } \\
\text { administración }\end{array}$ \\
\hline & $\begin{array}{l}\text { Administración } \\
\text { de empresas }\end{array}$ & $\begin{array}{l}\text { Cultura, patrimonio } \\
\text { e identidad }\end{array}$ & $\begin{array}{l}\text { Aspectos } \\
\text { funcionales }\end{array}$ & $\begin{array}{l}\text { Mercados } \\
\text { turísticos }\end{array}$ & Turismo y cultura \\
\hline & $\begin{array}{l}\text { Políticas y } \\
\text { normatividad }\end{array}$ & $\begin{array}{l}\text { Legislación, gestión } \\
\text { y políticas públicas }\end{array}$ & Superestructura & $\begin{array}{l}\text { Educación } \\
\text { superior }\end{array}$ & \\
\hline & $\begin{array}{l}\text { Diversa } \\
\text { vinculación } \\
\text { social }\end{array}$ & $\begin{array}{l}\text { Propuestas teórico- } \\
\text { conceptuales }\end{array}$ & $\begin{array}{l}\text { Epistemología, } \\
\text { conceptualización e } \\
\text { investigación }\end{array}$ & $\begin{array}{l}\text { Patrimonio y } \\
\text { gastronomía }\end{array}$ & \\
\hline & & & $\begin{array}{l}\text { Conducta, } \\
\text { motivación y } \\
\text { satisfacción del } \\
\text { turista }\end{array}$ & Demanda & \\
\hline & & & Mercadotecnia & $\begin{array}{l}\text { Comportamiento } \\
\text { del sector }\end{array}$ & \\
\hline & & & $\begin{array}{l}\text { Organización de la } \\
\text { comunidad }\end{array}$ & $\begin{array}{l}\text { Políticas } \\
\text { públicas }\end{array}$ & \\
\hline
\end{tabular}

Fuente: Elaboración propia.

existen trabajos que analizan particularmente el estado en que se encuentra el estudio de varios tópicos en la investigación turística. Pese a la importancia que cada uno de estos tópicos puede tener no solo en la construcción del conocimiento turístico nacional (y global) sino en sus implicaciones prácticas en las distintas realidades del país, esto no se ha hecho en los estudios turísticos en México. Por tanto, es menester realizar estudios muy focalizados en el desarrollo de temas concretos en el campo que reflejen en su conjunto 


\section{Monterrubio}

el avance de la investigación turística nacional. Llevar a cabo estudios detallados en torno a cómo se han abordado y qué tanto se han desarrollado tópicos específicos puede identificar nuevas oportunidades de investigación que permitan innovar líneas de investigación altamente especializadas, sobre todo entre los investigadores emergentes.

Aunque los rankings de investigadores y revistas en cualquier área del conocimiento suelen ser muy cuestionables, su necesidad es clara. Identificar a los investigadores y revistas "líderes", por denominarlos de alguna forma, es útil para entender cómo sus contribuciones lideran la construcción y las tendencias del conocimiento turístico (Zhao y Ritchie, 2007), tanto en el nivel general como particular (por tópico). A reserva de algunas excepciones (Picazo y Moreno, 2013), con sus particulares limitaciones, poco se ha hecho por identificar y analizar las contribuciones, liderazgo e impacto de académicos y revistas en la investigación turística en México.

En comparación con el marco internacional, la cantidad de revistas en donde los investigadores mexicanos suelen publicar es menor (Picazo y Moreno, 2013). De este modo, otra necesidad presente en el análisis del estado que guarda la investigación turística en el país es precisamente estudiar las aportaciones y el impacto científico tanto de investigadores del país como de las revistas en donde suelen publicar. Esto permitiría no solo un análisis más incluyente y exhaustivo de los avances de la investigación turística en el país, al poder integrar a un número considerable y quizás representativo de unidades de análisis, sino también un estudio detallado de las particularidades, tanto cuantitativas como cualitativas, de tal liderazgo. Un seguimiento más preciso sobre las contribuciones por investigador, institución y revistas puede llevar a resultados más confiables en cuanto al verdadero impacto de académicos y revistas científicas en la investigación turística en México.

No obstante que en el marco de la investigación turística internacional existen varias oportunidades y necesidades para el caso mexicano, desde la perspectiva del autor hay una necesidad muy importante que aún se encuentra pendiente. El papel de las revistas internacionales, específicamente aquellas del contexto anglosajón, en la construcción, diseminación, discusión y comunicación de la investigación turística en el mundo es muy relevante; muestra de ello es el índice de citación e influencia de estas en distintos idiomas (Pearce, 2013). Estas revistas no solo concentran trabajos hechos por, y en el contexto de, países anglosajones; a esta construcción se han sumado "conocimientos locales" de otros entornos y países, que han contribuido también a partir de sus propias particularidades al debate del conocimiento en el área. En consecuencia, lo que hoy en día pudiera ser entendido como la investigación turística internacional, si bien dominada por académicos de países anglosajones, no representa únicamente el conocimiento de los llamados países industrializados, puesto que también ha sido matizada por aportaciones de otros contextos, aunque estas componen la gran minoría.

De esta forma, el "conocimiento local” de las realidades turísticas de México ha permanecido ampliamente fuera del discurso internacional, o al menos de aquellas publicaciones reconocidas no solo en el mundo anglosajón, sino en muchas otras. Dada la variada 
actividad turística del país, este tiene mucho que aportar al cuerpo de conocimientos y debates en la investigación turística en el mundo. Mientras que las contribuciones de México al conocimiento internacional podrían ser consideradas una oportunidad de investigación para los estudiosos nacionales, estas son, en la mirada del autor, una necesidad en la investigación turística internacional.

\section{Conclusiones}

En sus distintas dimensiones, los avances en la investigación turística como tal han sido objeto de estudio. Tanto en el nivel internacional como en países específicos, incluyendo México, las tendencias y los estados que guarda esta investigación en términos conceptuales, teóricos, metodológicos y de liderazgo en autoría, han sido analizados, independientemente de la forma en que esto se ha realizado. Si bien los esfuerzos al respecto han sido indicativos de tales tendencias y estados, es necesario tener en cuenta que, debido a que la investigación turística constituye un ente diferenciable en su interior por ser multitemática, multicontextual, multidisciplinaria y abordada de modo distinto en cuanto a tiempos, espacios, temas, conceptos, teorías, metodologías y autores, difícilmente se pueden hacer precisiones sobre sus avances generales, no solo entre diferentes naciones sino dentro de ellas.

Como en otros países, la investigación turística en México ha sido también abordada como objeto de estudio, aunque aún de manera limitada. Este artículo revela que se han hecho distintos estudios sobre algunos avances de esta investigación en el país. Más allá de las coincidencias o disidencias que los estudios existentes han mostrado, hay vacíos dentro de la investigación turística en México que requieren atención académica en el marco de la investigación turística internacional. Al hacer una evaluación del estado y las tendencias del campo se observa, por un lado, que los trabajos de investigación en el país han sido muy limitados temáticamente, lo que exige una ampliación de los tópicos estudiados atendiendo a los diferentes alcances del turismo como un fenómeno multidimensional, y esto a su vez permitirá una comprensión más integral del fenómeno.

Por otro lado, se concluye que poco se conoce en torno a los avances temáticos, teóricos, conceptuales y metodológicos más recientes del campo de estudio del turismo como un todo. Específicamente existe una necesidad de conocer tales avances desde metodologías más incluyentes de los distintos indicadores de la investigación turística; se requiere adoptar metodologías y perspectivas que hagan posible una contemplación más amplia de las fuentes, los contenidos y otros elementos indicadores de la investigación en el país. Los análisis bibliométricos, en particular los análisis del conocimiento publicado en revistas especializadas, necesitan incorporarse a la agenda de la investigación turística nacional.

Asimismo, para conocer con mayor profundidad los avances hasta hoy logrados en México, la comunidad académica requiere hacer análisis más dirigidos acerca de tópicos específicos en el turismo; qué conceptos han emergido, qué posturas teóricas y metodológicas se han venido desarrollando, qué utilidad práctica están teniendo los estudios 


\section{Monterrubio}

turísticos en el país, son preguntas de investigación que quedan pendientes; así como la necesidad de examinar en dónde, cómo y quiénes están produciendo y concentrado la mayor parte de la investigación turística en México, es decir, se precisan estudios que revelen quiénes y de qué forma están liderando, si es así, la construcción del conocimiento en el campo.

Por último, una necesidad latente ante la investigación turística es incorporar el conocimiento específico de México basado en sus experiencias, paradigmas y realidades particulares al corpus del conocimiento internacional. Integrar los "conocimientos locales" de México, y de muchos otros países que no han formado parte del debate del conocimiento turístico internacional, permitirá tener un entendimiento más amplio del turismo como actividad y campo de estudio global.

Al igual que cualquier otro estudio, las reflexiones y necesidades aquí vertidas deben ser tomadas con cautela. Se debe considerar que, debido a que se trata principalmente de un estudio en gran parte fundamentado en información de segunda mano, esto es, en trabajos que han analizado por su propia cuenta los avances de la investigación, las ideas del presente están ceñidas a los alcances de tal información. Seguramente, si se utilizaran otras fuentes de análisis, las reflexiones y conclusiones serían distintas, lo cual no significa por supuesto que las conclusiones de este trabajo sean inválidas o incompletas, sino que otras aproximaciones de análisis brindarían una complementariedad valiosa para el objetivo aquí perseguido. Esto ofrece, por tanto, la oportunidad de realizar análisis y propuestas similares desde otras ópticas metodológicas, incluyendo el análisis de datos primarios.

\section{Referencias}

Albacete-Sáez, C. y Fuentes-Fuentes, M. M. (2010). Difusión de la investigación española sobre turismo en revistas internacionales. Revista de Análisis Turístico, 9(1), 14-29.

Ashworth, G. y Page, S. J. (2011). Urban tourism research: Recent progress and current paradoxes. Tourism Management, 32(1), 1-15.

Ballantyne, R., Packer, J. y Axelsen, M. (2009). Trends in tourism research. Annals of Tourism Research, 36(1), 149-152.

Bao, J., Chen, G. y Ma, L. (2014). Tourism research in China: Insights from insiders. Annals of Tourism Research, 45(1), 167-181.

Bushell, R., Prosser, G. M., Faulkner, H. W. y Jafari, J. (2001). Tourism research in Australia. Journal of Travel Research, 39(3), 323-326.

Castellucci, D. (2001). Diagnóstico del estado de la investigación turística en las universidades argentinas. APORTES y Transferencias, 5(1), 95-111.

Castillo, M. y Lozano, M. (2006). Apuntes para la investigación turística. Chetumal: Universidad de Quintana Roo.

Cestur (2007). Elaboración de un diagnóstico del estado de la investigación turística en el país y generación de un programa de investigación integral para el sector turismo. México: Secretaría de Turismo. 
Cheng, C.-K., Li, X. (R.), Petrick, J. F. y O'Leary, J. T. (2011). An examination of tourism journal development. Tourism Management, 32(1), 53-61.

Dann, G. (1981). Tourist motivation. An appraisal. Annals of Tourism Research, 8(2), 187219.

Decrop, A. (1999). Triangulation in qualitative tourism research. Tourism Management, 20(1), 157-161.

Deery, M., Jago, L. y Fredline, L. (2012). Rethinking social impacts of tourism research: A new research agenda. Tourism Management, 33(1), 64-73.

Espinosa, M. (2007). La investigación turística en México: Tendencias y retos. En M. Espinosa (ed.), Tendencias de investigación turística a principios del siglo XxI (pp. 17-46). México: Instituto Politécnico Nacional.

Figueroa-Domecq, C., Pritchard, A., Segovia-Pérez, M., Morgan, N. y Villacé-Molinero, T. (2015). Tourism gender research: A critical accounting. Annals of Tourism Research, 52(1), 87-103.

Galani-Moutafi, V. (2004). Tourism research on Greece. A critical overview. Annals of Tourism Research, 31(1), 157-179.

Goeldner, Ch. (2005). Reflections on the historic role of journals in shaping tourism knowledge. Journal of Tourism Studies, 16(2), 44-51.

Guevara, R., Molina, S. y Tresserras, J. (2006). Hacia un estado de la cuestión en investigación turística. En R. Guevara (ed.), Estudios multidisciplinarios en turismo (pp. 17-68). México: Secretaría de Turismo.

Gursoy, D. y Sandstrom, J. K. (2016) An updated ranking of hospitality and tourism journals. Journal of Hospitality and Tourism Research, 40(1), 3-18.

Hall, C. M. (2011). Publish and perish? Bibliometric analysis, journal ranking and the assessment of research quality in tourism. Tourism Management, 32(1), 16-27.

Hiernaux, D. (2000). Los nuevos derroteros de la investigación turística. En N. Monterroso y L. Zizumbo (eds.), Investigación turística (pp. 39-56). Toluca: Universidad Autónoma del Estado de México.

Jiménez, A. y Sosa, A. (2006). Notas sobre las etapas de la investigación del turismo en México. En Secretaría de Turismo (ed.), Análisis del turismo (pp. 59-76). México: Secretaría de Turismo.

Jiménez, A., Osorio, M. y Avila, R. M. (2006). Caracterización general del listado de la Red de Investigadores y Centro de Investigación en Turismo (RICIT). En VIII Congreso Nacional y 20 Internacional de Investigación Turística. Monterrey, 14-16 de junio de 2006. [Documento original proporcionado por el primer autor.]

Johnson, P. y Thomas, B. (1990). Employment in tourism: A review. Industrial Relations Journal, $21(1), 36-48$.

$\mathrm{Li}, \mathrm{L}$. (2008). A review of entrepreneurship research published in the hospitality and tourism management journals. Tourism Management, 29(6), 1013-1022.

McKercher, B. (2008). A citation analysis of tourism scholars. Tourism Management, 29(6), 1216-1232.

McKercher, B. (17 de septiembre de 2013). Academic journals of tourism, hospitality and events. En Tourism Research Information Network (TRINET).

McKercher, B. y Tung, V. (2015). Publishing in tourism and hospitality journals: Is the past a prelude to the future? Tourism Management, 50, 306-315. 


\section{Monterrubio}

McKercher, B., Law, R. y Lam, T. (2006). Rating tourism and hospitality journals. Tourism Management, 27(6), 1235-1252.

Osorio, M., Castillo, M. y Moreno, I. (2007). Investigación y conocimiento turístico en México. Un acercamiento a la contribución de las instituciones de educación superior y centros de investigación (IES y CIS). En C. Virgen (ed.), Aportaciones de investigación turística (pp. 29-48). Guadalajara: Universidad de Guadalajara.

Pearce, D. G. (2013). The internationalization of tourism. Journal of Travel Research, 53(3), 267-280.

Pechlaner, H., Zehrer, A., Matzler, K. y Abfalter, D. (2004). A ranking of international tourism and hospitality journals. Journal of Travel Research, 42(4), 328-332.

Phillimore, J. y Goodson, L. (2004). Progress in qualitative research in tourism: Epistemology, ontology and methodology. En J. Phillimore y L. Goodson (eds.), Qualitative Research in Tourism: Ontologies, Epistemologies and Methodologies (pp. 3-29). Londres: Routledge.

Picazo, P. y Moreno, S. (2013). Difusión de la investigación científica en turismo. El caso de México. El Periplo Sustentable, 24, 7-40.

Pike, S. (2002). Destination image analysis - a review of 142 papers from 1973 to 2000. Tourism Management, 23(5), 541-549.

Riley, R. y Love, L. L. (2000). The state of qualitative tourism research. Annals of Tourism Research, 27(1), 164-187.

Ryan, Ch. (2005). The ranking and rating of academics and journals in tourism research. Tourism Management, 26(5), 657-662.

Schlüter, R. y Bertoncello, R. (2010). Tourism research in Latin America: Past and future challenges. En D. G. Pearce y R. W. Butler (eds.), Tourism Research: A 20-20 Vision (pp. 2-13). Oxford: Goodfellow Publishers Limited.

Sharpley, R. (2011). The Study of Tourism. Past Trends and Future Directions. Londres: Routledge.

Sharpley, R. (2014). Host perceptions of tourism: A review of the research. Tourism Management, 42(1), 37-49.

Sheldon, P. J. (1990). Journals in tourism and hospitality. The perceptions of publishing faculty. The Journal of Tourism Studies, 1(1), 42-48.

Sheldon, P. J. (1991). An authorship analysis of tourism research. Annals of Tourism Research, 18(3), 473-484.

Song, H., Dwyer, L., Li, G. y Cao, Z. (2012). Tourism economics research: A review and assessment. Annals of Tourism Research, 39(3), 1653-1682.

Swain, M. B., Brent, M. y Long, V. H. (1998). Annals and tourism evolving: Indexing 25 years of publication. Annals of Tourism Research, 25(Supplement), 991-1014.

Tamayo, A. L. y Peñaloza, L. (2005). Investigación en turismo y educación en la Universidad Autónoma del Estado de México. Teoría y Praxis, 1, 87-95.

Tribe, J. y Xiao, H. (2011). Developments in tourism social science. Annals of Tourism Research, 38(1), 7-26.

Walle, A. H. (1997). Quantitative versus qualitative tourism research. Annals of Tourism Research, 24(3), 524-536.

Weed, M. (2006). Sports tourism research 2000-2004: A systematic review of knowledge and a meta-evaluation of methods. Journal of Sport \& Tourism, 11(1), 5-30. 
Weed, M. (2009). Progress in sports tourism research? A meta-review and exploration of futures. Tourism Management, 30(5), 615-628.

Xiao, H. y Smith, S. L. J. (2006a). Case studies in tourism research: A state-of-theart analysis. Annals of Tourism Research, 27(5), 738-749.

Xiao, H. y Smith, S. L. J. (2006b). The making of tourism research: Insights from a social science journal. Annals of Tourism Research, 33(2), 490-507.

Xiao, H., Jafari, J., Cloke, P. y Tribe, J. (2013). Annals: 40-40 vision. Annals of Tourism Research, 40(January), 352-385.

Xin, S., Tribe, J. y Chambers, D. (2013). Conceptual research in tourism. Annals of Tourism Research, 41(1), 66-88.

Zhao, W. y Ritchie, J. R. B. (2007). An investigation of academic leadership in tourism research: 1985-2004. Tourism Management, 28(2), 476-490. 